\title{
Running-in Behavior in the Combination of Diamond-Like Carbon Film and AC8A Aluminum Alloy
}

\author{
Tomomi Honda ${ }^{1)^{*}}$, Toshiyuki Nagai ${ }^{1)}$, Toshiro Miyajima ${ }^{2)}$, Yoshiro Iwai ${ }^{1)}$, \\ Takatoshi Shinyoshi ${ }^{3)}$ and Yoshio Fuwa ${ }^{4)}$ \\ ${ }^{1)}$ Graduate School of Engineering, University of Fukui, 3-9-1 Bunkyo, Fukui, Fukui 910-8507, Japan \\ ${ }^{2)}$ Department of Mechanical Systems Engineering, Toyama Prefectural University, \\ 5180 Kurokawa, Imizu, Toyama 939-0398, Japan \\ ${ }^{3)}$ Toyota Motor Co. Ltd., 1, Toyota-cho, Toyota, Aichi 471-8572, Japan \\ ${ }^{4)}$ Fuwa Technical Office, 7-167, Miyukihonmachi, Toyota, Aichi 473-0901, Japan \\ *Corresponding author: Tomomi Honda (honda@u-fukui.ac.jp)
}

Manuscript received 05 June 2018; accepted 03 September 2018; published 30 September 2018

\begin{abstract}
The running-in process affects the friction and wear properties of sliding materials in machines. If it is not done correctly, serious problems such as machine seizure may occur. Diamond-like carbon (DLC) films are used in various fields as an excellent tribo-material because of their low friction coefficient and high wear resistance. However, their running-in behavior has not been clarified quantitatively in detail. In addition, previous studies have not adequately investigated changes in wear particles during the running-in process. In the present work, we investigated the correlation between the friction coefficient and the number of wear particles during the runningin process using an online particle counter. Then, we compared the sliding distance at the completion of running-in judged using the particle counter with that judged using the friction coefficient. As a result, the running-in behavior in the combination of a DLC film and AC8A Al alloy is well clarified. In the case of a DLC/AC8A sliding pair, the DLC surface showed no significant changes after wear tests. The AC8A surface became smooth by abrasion and plastic deformation during running-in, and the ratio of silicon occupying the surface area increased slightly.
\end{abstract}

\section{Keywords}

running-in, diamond-like carbon, AC8A aluminum alloy, particle counter, friction coefficient

\section{Introduction}

The running-in process for sliding parts in machines has long been known to be important because it affects the friction and wear properties of the sliding materials. Runningin procedures are often empirically determined, as there is little prior knowledge of how the sliding surface will actually respond to running-in [1, 2]. If running-in is not done correctly, serious problems such as machine seizure may occur. However, running-in behavior is not well clarified quantitatively because the running-in process is complex and involves various factors. For instance, the formation of oxidation films on the wear surface, decrease in the plasticity index, an increase in surface hardness, and improvement of wear and seizure resistance by a tribo-layer must be considered [3, 4]. In addition, previous studies have not adequately investigated changes in the wear particles during running-in. The characteristics of wear particles carried in the flow of circulating oil represent the lubricant conditions at rubbing surfaces [5]. In a previous study, the authors have developed a new technique that can estimate the wear amount quantitatively under lubricated conditions using the on-line particle counter. The authors reported the possibility of a quantitative diagnosis of rubbing surface failure [6].

Recently, diamond-like carbon (DLC) films have been applied in various fields as an excellent tribo-material because of their low friction coefficient, high wear resistance, and chemical inertness. AC $8 \mathrm{~A} \mathrm{Al}$ alloy is expected to apply to the engine for automobiles because of their low density and good wear resistance from the viewpoints of reducing fuel consumption.

In this study, wear tests were performed by rubbing the annular surface of a ring (AC8A) against a fixed plate (DLC) under lubrication. The number of wear particles was measured using an online particle counter and a correlation with the friction coefficient was found. We discuss the running-in behavior in the combination of the DLC film and AC8A aluminum alloy. 


\section{Experimental}

\subsection{Test materials}

Table 1 shows the material properties of both specimens. Not only the roughness but also the waviness will affect to the fluid pressure generation under lubrication and the distribution of the real contact area. But, it does not take into consideration in this paper. The surface was finished as flatly as possible using lapping machine. The DLC films, namely the amorphous hydrogenated carbon coating, a-C:H, were deposited on a bearing material JIS SUJ2 substrate by plasma chemical vapor deposition (CVD). Hydrogen content is $16 \%$, and ID/IG ratio is 0.51 . The thickness of the DLC film, which was measured using a ball crater method, was approximately 2 $\mu \mathrm{m}$. Its hardness, which was measured using a nano-indention tester, was 23.5 GPa. The indentation hardness was measured at seven randomly distributed points under a load of $4.9 \mathrm{mN}$. The indentation depth doesn't exceed $0.2 \mu \mathrm{m}$. The hardness is the mean value of five points except the maximum value and minimum value. The nano-indention tester has the trigonal diamond indenter with a vertical angle of $115^{\circ}$.

The ring was an aluminum-silicon alloy. This material is designated as AC8A in the Japan Industrial Standard. The silicon had eutectic structure and was present at 12 mass $\%$. The Vickers hardness was measured at seven randomly distributed points under a load of $0.98 \mathrm{~N}$. It is the mean value of five points except the maximum value and minimum value. The Vickers hardness tester has the pyramidal diamond indenter with a vertical angle of $136^{\circ}$.

\subsection{Test apparatus and procedure}

The experiments were performed with a ring-on-plate test rig. Figure 1 shows a schematic view of the test apparatus. Two particle counters (designated as IN PC and OUT PC, respectively) were set along the pipe in which the lubricating oil circulated from an oil purifier to the test apparatus and from the test apparatus to an external tank. Flow rate of sample fluid

Table 1 Material properties

\begin{tabular}{c|c|c}
\hline \multirow{2}{*}{ Material } & Plate & Ring \\
\cline { 2 - 3 } & DLC & AC8A \\
\hline Vickers hardness & - & 89.9 \\
\hline Indentation hardness, $\mathrm{GPa}$ & 23.5 & - \\
\hline Surface roughness $\mathrm{R} a, \mu \mathrm{m}$ & 0.02 & 0.11 \\
\hline
\end{tabular}

is $300 \mathrm{ml} / \mathrm{min}$. So, after generating the wear particles, we can measure the size and distribution of them at once. And, we can estimate the wear amount quantitatively [6]. Wear tests were performed by rubbing the annular surface of a ring (AC8A) against a fixed plate (DLC) at applied loads of $200 \mathrm{~N}, 800 \mathrm{~N}$, and $1400 \mathrm{~N}$ and a sliding velocity of $2.0 \mathrm{~m} / \mathrm{s}$. The sliding distance was $10 \mathrm{~km}$.

Wear tests were performed under lubrication. The sliding surface was immersed in an oil bath filled with lubricating oil at a temperature of about $60 \pm 1^{\circ} \mathrm{C}$. The lubricating oil is nonadditive paraffinic mineral oil of group II for industrial use. The oil viscosity at $60^{\circ} \mathrm{C}$ was $14.8 \mathrm{~mm}^{2} / \mathrm{s}$. The lubrication mode was the mixed lubrication. The friction torque of the rotating table on which the plate specimen was mounted was interrupted by a load cell attached to the rigid body. Its friction torque was measured, and then the friction coefficient was calculated.

\subsection{Particle counter}

The outline is described here because details concerning the structure and the measurement accuracy of the particle counter have been reported in the references [5] and [6]. Figure 2 shows a schematic view of the particle counter. Selfoc lenses of 0.25 $\mathrm{P}$ (pitch) and $0.5 \mathrm{P}$ are set in front of a laser diode (LD) and a photodetector $(\mathrm{PD})$, respectively, to guide the laser beam to the sensor zone. The laser beam is detected by the PD thorough a small square slit $(50 \mu \mathrm{m} \times 50 \mu \mathrm{m})$.

Figure 3 shows a schematic of the signal treatment. When a particle crosses the sensor zone, the output voltage of the PD drops in proportion to the projected area of the particle as shown in Fig. 3(a). The pulse goes through a high-pass filter and

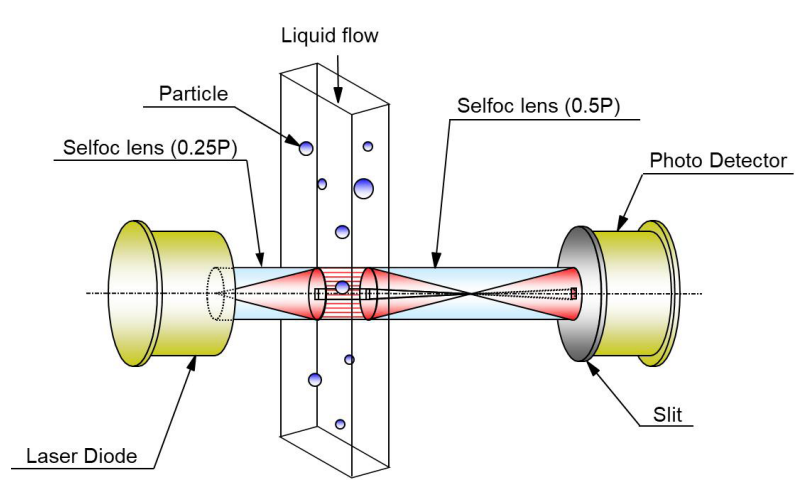

Fig. 2 Schematic of the particle counter

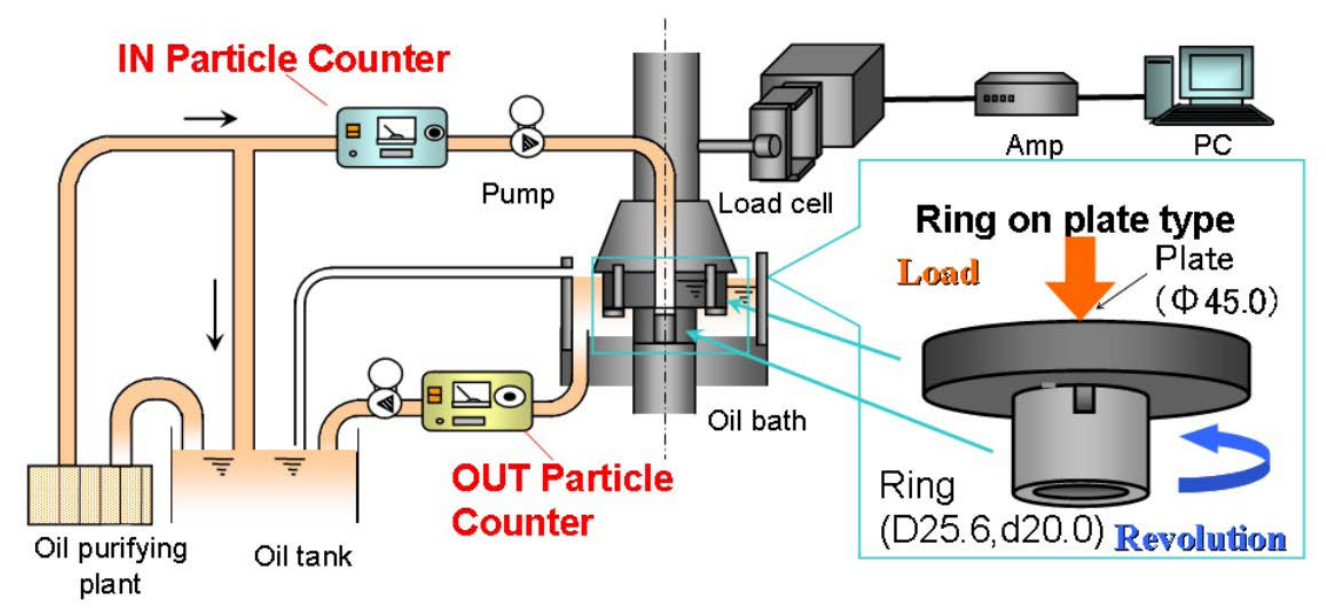

Fig. 1 Schematic of the wear test apparatus 


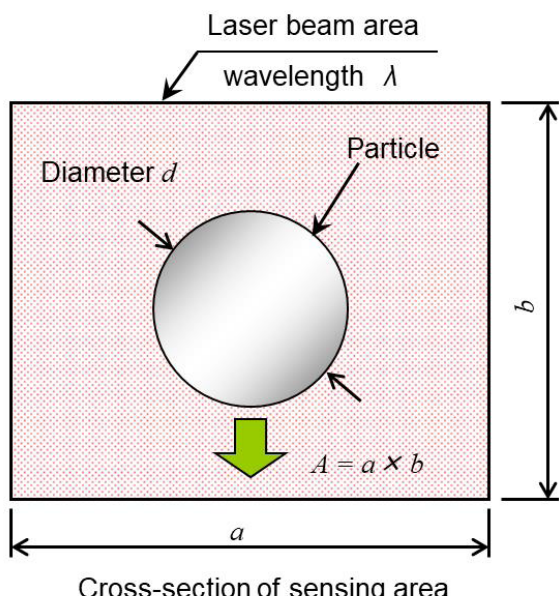

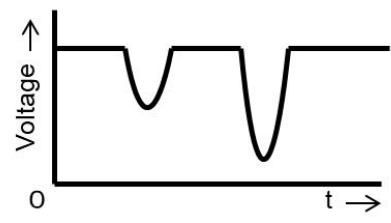

(a) Output of photo detector

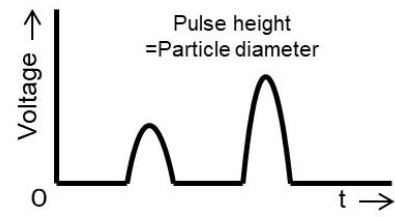

(b)Output of high pass filter Signal pulse shapes

Fig. 3 Schematic of the signal treatment

is then inverted as shown in Fig. 3(b). The peak of the pulse is detected by an $\mathrm{A} / \mathrm{D}$ converter and counted by a signal processor. The pulse height is converted into the diameter of an equivalent circle for the projected area [5]. The specifications of the particle counter are as follows. Minimum detectable diameter of particles is $5 \mu \mathrm{m}$, maximum concentration of particles is 100,000 counts $/ \mathrm{ml}$, the color is ASTM- 8 grade, temperature of sample fluid is $0-80^{\circ} \mathrm{C}$, and number of particles are counted with an interval of $1 \mu \mathrm{m}$.

\section{Results and discussion}

3.1 Variations in the friction coefficient and the number of wear particles

Figure 4 shows the friction coefficient and the number of wear particles at various applied loads. Both of these values decreased rapidly in the initial stage and then gradually decreased to a steady-state value. The initial friction coefficient was around 0.3 ; it rapidly decreased to $0.02-0.03$. The friction coefficient decreased slightly as the applied load increased. The number of wear particles was around $100-200$ initially and then decreased to 50 . The number of wear particles increased with the applied load. In terms of the characteristic changes in the friction coefficient and the number of wear particles as a function of sliding distance, both values showed the similar tendency. They gradually decreased with the sliding distance, and then they became constant.

\subsection{Comparison of the running-in completion distance}

Figure 5 illustrates how the running-in completion distance is defined. The variations in the friction coefficient and the number of wear particles as a function of sliding distance are approximated by the ninth function using the least square method. When the gradient of friction coefficient vs. sliding distance is less than $10^{-1}$ and that of the number of wear particles vs. the sliding distance is less than $10^{-3}$, we defined the distance as the running-in completion distance. The distances defined by both parameters are almost constant and stable.

The two values are denoted by $d_{p}$ (running-in completion distance judged by the number of wear particles) and $d_{\mu}$ (that judged by the friction coefficient). Figure 6 compares the two running-in completion distances. The results show good correlation between $d_{p}$ and $d_{\mu}$ in the running-in process. The results of the number of wear particles using the particle counter apprehend the running-in process well. (a)

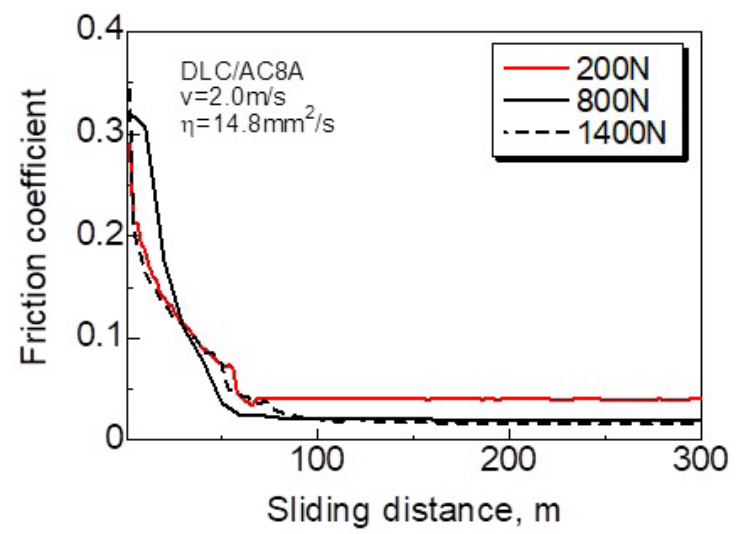

(b)

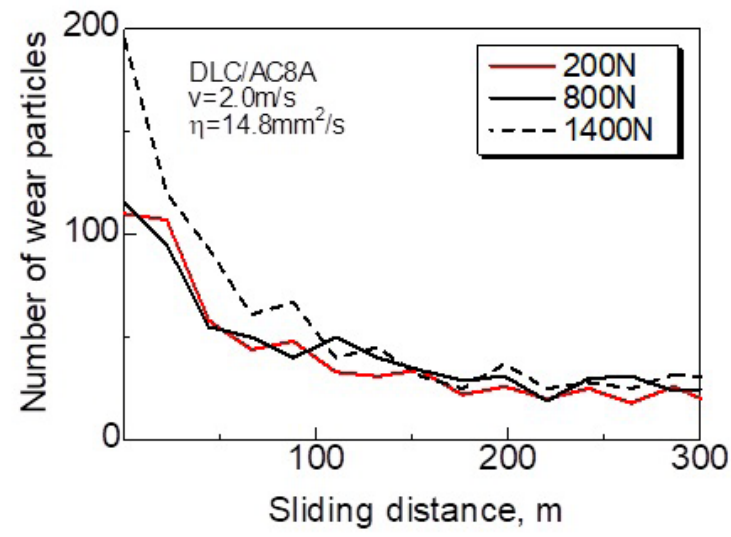

Fig. 4 Variations in friction coefficient and number of wear particles at various contact loads

(a) Friction coefficient, (b) Number of wear particles

\subsection{Wear surface}

After wear tests, the worn surfaces of the plate and ring specimens were observed using an optical microscope and a scanning electron microscope. In addition, the worn surfaces were analyzed using an energy dispersive X-ray spectrometry (EDX). Figure 7 shows optical microscope images and crosssectional profiles of both specimens. The worn surface of the DLC plate showed no significant differences at sliding distances of $80 \mathrm{~m}, 130 \mathrm{~m}$, and $10 \mathrm{~km}$. Figure 8 shows the EDX analysis of the worn DLC surface at various sliding distances. The EDX chemical analysis shows that aluminum is slightly transferred from the ring to the plate at sliding distance of $80 \mathrm{~m}, 130 \mathrm{~m}$ and $10 \mathrm{~km}$. 
The worn surface of the AC8A ring became smooth by wear and plastic deformation. In the combination of diamond-like carbon film and AC8A aluminum alloy, the aluminum matrix is worn by a small-scale adhesive wear as priority and a lot of Si distributes on the sliding surface. So, surface strength increases and wear decreases. Then the running-in is finished. We observed the wear particles in the sliding distance of $80 \mathrm{~m}$. Al wear particles in the scale of 5-10 $\mu \mathrm{m}$ were observed as shown in Fig. 9 .

The surface profiles indicate that smoothing during running-in is more significant in the ring than in the plate. In the process of smoothing, the surface layer becomes harder than

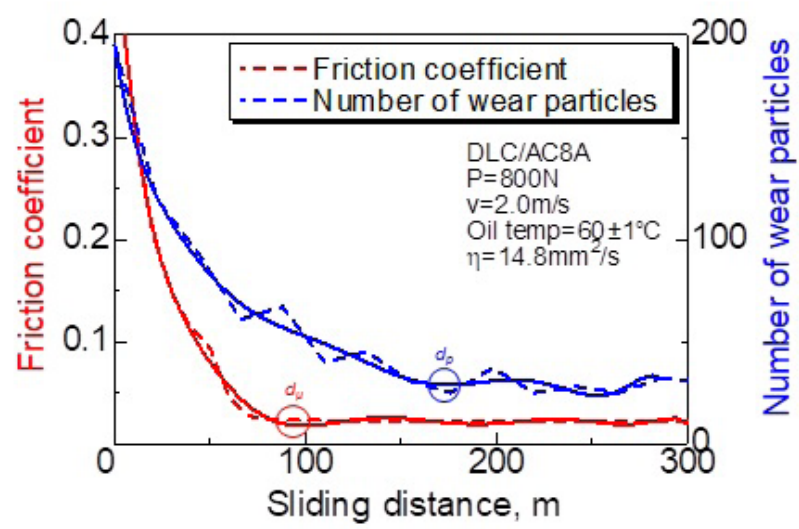

Fig. 5 Definition of running-in distance

(a)

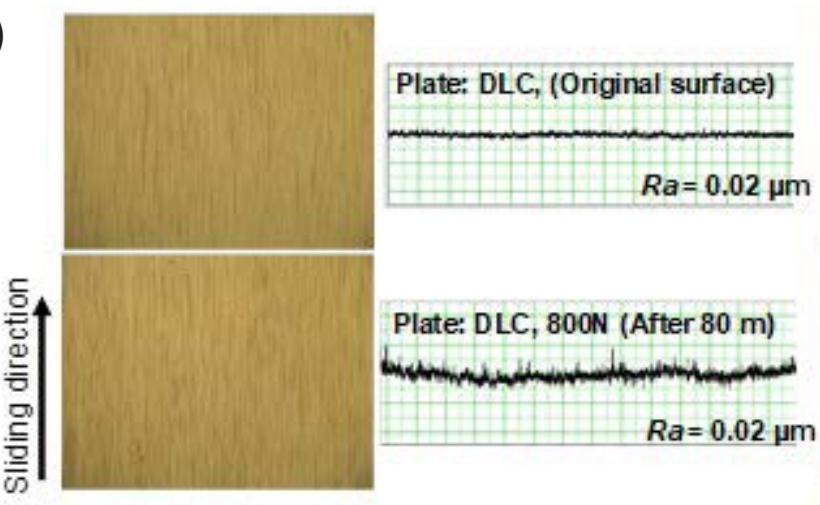

(b)
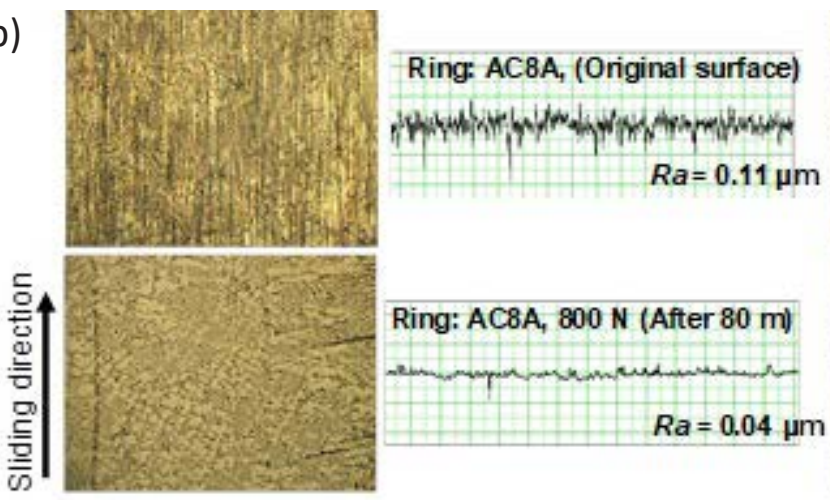

that of the as-received material [7]. The presence of a highly deformed surface layer may explain why rough surfaces are generally believed to run in more successfully [8]. Again, the surface profile changed within the first minutes of sliding. We guessed that the contact between asperities on the sliding surface decreased and the contact pressure decreased as the

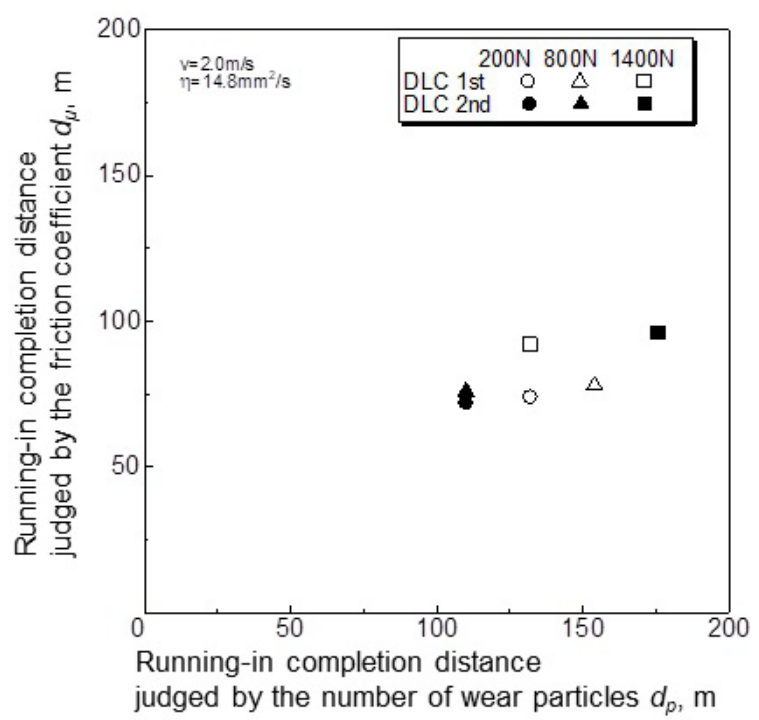

Fig. 6 Comparison of the running-in completion distances
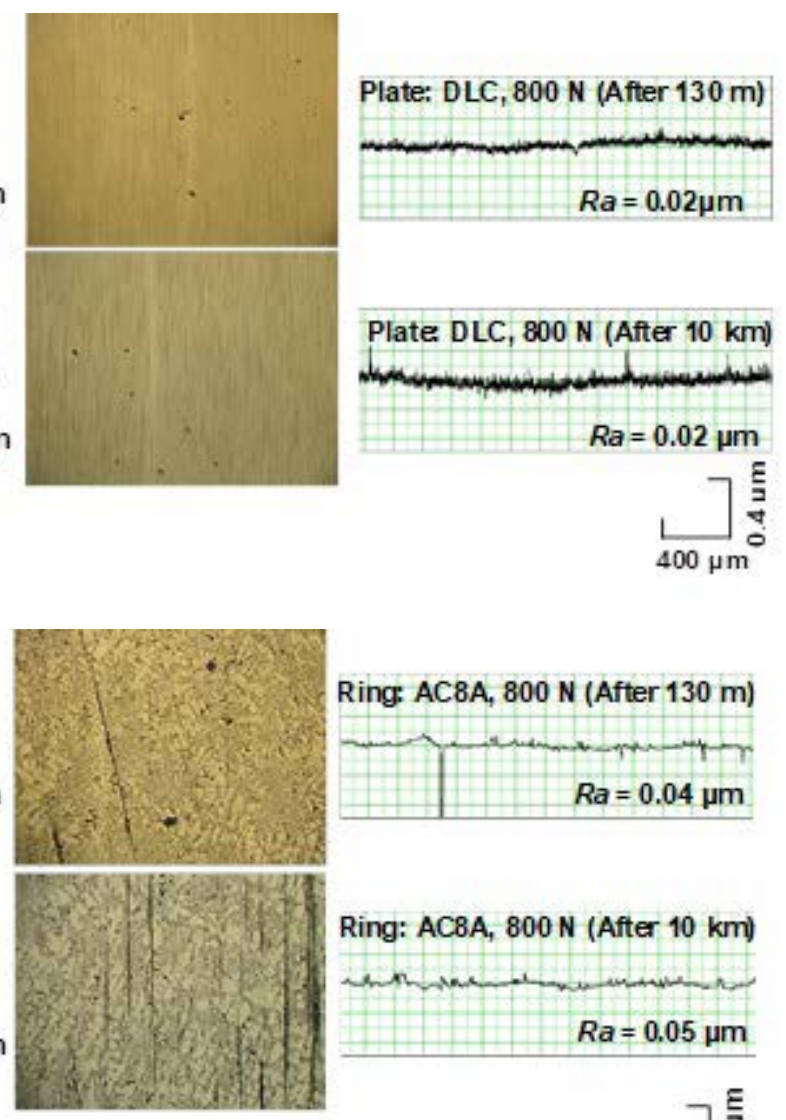

Ring: AC8A, $800 \mathrm{~N}$ (After $130 \mathrm{~m}$ )

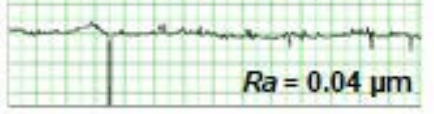

Ring: AC8A, $800 \mathrm{~N}$ (After $10 \mathrm{~km}$ )

$R a=0.05 \mu \mathrm{m}$

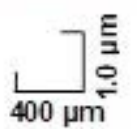

Fig. 7 Optical microscope images and cross-sectional profiles of the specimens

(a) Plate specimen: DLC, (b) Ring specimen: AC8A 

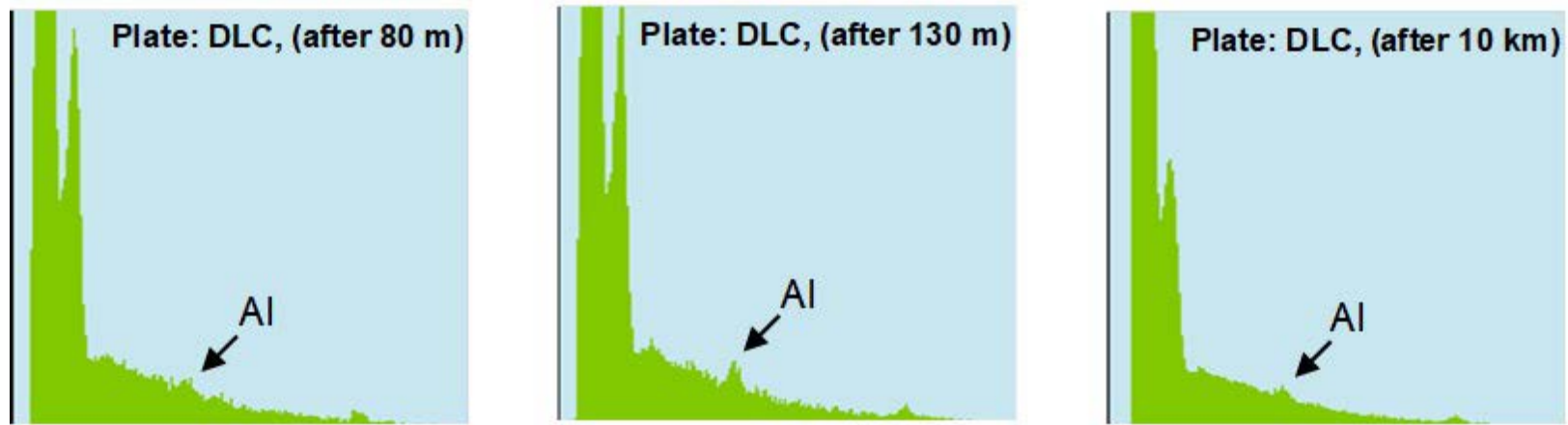

Fig. 8 EDX analysis of the worn surface of DLC films at various sliding distances
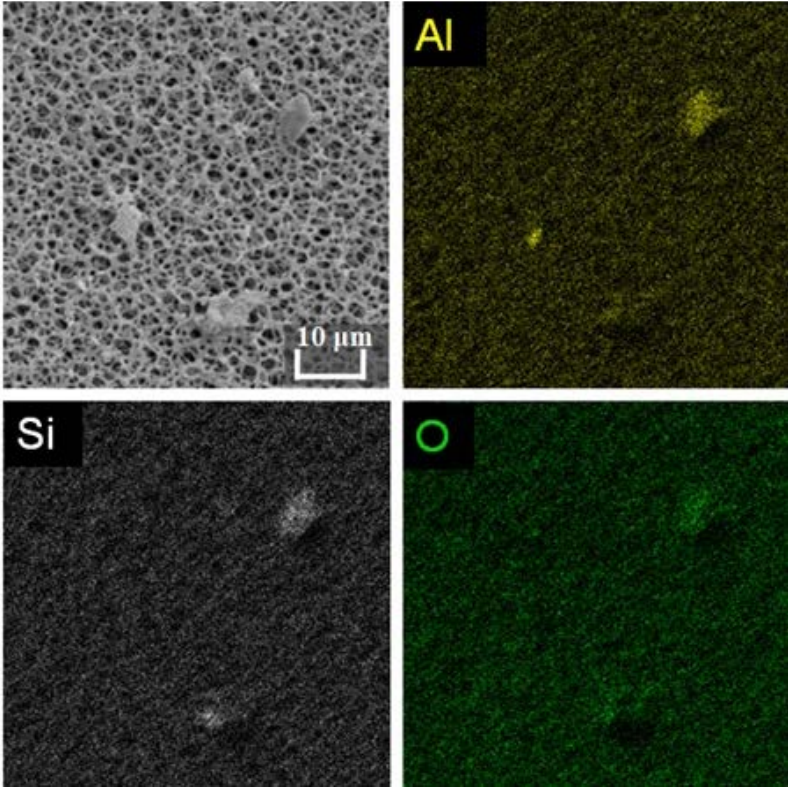

Fig. 9 SE image and EDX images of wear particles at a sliding distance of $80 \mathrm{~m}$

worn surface of AC8A became smooth, that an oil film was gradually created at the interface.

Figure 10 shows EDX images of the AC8A surface at various sliding distances. The ratio of silicon occupying the surface area increased slightly at $80 \mathrm{~m}\left(d_{\mu}\right)$, as well as at $150 \mathrm{~m}$ and 10 $\mathrm{km}$. From these results, we expect that wear of the AC8A was limited.

\section{Conclusion}

(1) A particle counter may be able to use to determine the running-in completion distance from the results of the correlation between the friction coefficient and the number of wear particles during running-in.

(2) In the case of a DLC/AC8A sliding pair, the DLC surface showed no significant changes after wear testing. The AC8A surface became smooth by abrasion and plastic deformation during running-in, and the ratio of silicon occupying the surface area increased slightly.

(3) The friction coefficient decreased slightly and the number of wear particles increased with an increase of applied load.

\section{References}

[1] Cavatorta, M. P. and Cusano, C., “Running-in of Aluminum/Steel

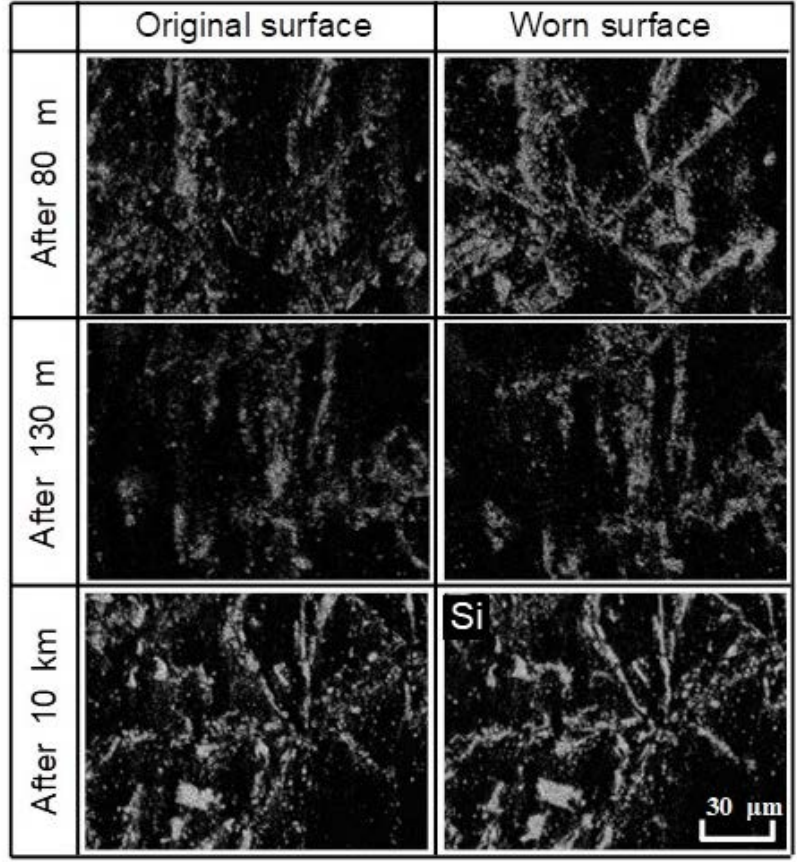

Fig. 10 EDX images of AC8A (ring) at various sliding distances. White area means the existence of $\mathrm{Si}$

Contacts under Starved Lubrication: Part I: Surface Modifications," Wear, 242, 1-2, 2000, 123-132.

[2] Katoh, J., Mizuhara, K., Satou, T. and Kamikubo, F., "Analytical Techniques on the Running-in Process of Sliding Wear," Kobe Steel Engineering Reports, 50, 2, 2000, 66-69 (in Japanese).

[3] Sato, H., Kyogoku, K., Chao, S. and Nakahara, T., "Estimation of Running-in Process by Means of Friction Patterns," Journal of Japan Society of Lubrication Engineers, 31, 2, 1986, 102-109 (in Japanese).

[4] Hirano, F., "Running-in Process of Metals," Journal of Japan Society of Lubrication Engineers, 30, 5, 1985, 377-384 (in Japanese).

[5] Yoshinaga, S., Iwai, Y., Nishino, E., Nishizawa, H. and Kobayashi, T., "Real-Time Counting Method of Small Particle Size Distribution Using a Laser Diode," Kogaku, 26, 5, 1997, 273-279 (in Japanese).

[6] Iwai, Y., Honda, T., Miyajima, T., Yoshinaga, S., Higashi, M. and Fuwa, Y., "Quantitative Estimation of Wear Amounts by Real Time Measurement of Wear Debris in Lubricating Oil," Tribology International, 43, 1-2, 2010, 388-394

[7] Blau, P. J., "Friction and Wear Transitions of Materials," Noyes Publications, NJ, 1989.

[8] Sreenath, A. V. and Raman, N., "Running-in Wear of a Compression Ignition Engine: Factors Influencing the Conformance between Cylinder Liner and Piston Rings," Wear, 38, 2, 1976, 271-289. 